\title{
Ultrafast MRI in the prenatal diagnosis of Bourneville's tuberous sclerosis
}

\author{
P. C. Khanna, S. Godinho, S. A. Pungavkar, D. P. Patkar \\ Department of Magnetic Resonance Imaging, Dr. Balabhai Nanavati Hospital, Vile Parle, Mumbai, India
}

\begin{abstract}
The purpose of this report is to highlight the utility of prenatal MRI as an adjunctive imaging modality in the diagnosis and prognosis of Tuberous Sclerosis (TS) (Bourneville's disease). We report a case of TS detected in utero at 30 weeks gestation. A routine ultrasonography at 26 weeks in a $28-$ year-old primigravida was followed by an ultrafast MRI examination at 30 weeks gestation. Ultrasound raised the possibility of TS based on the detection of multiple cardiac rhabdomyomas. Fetal MRI, subsequently performed, showed the presence of cortical tubers and subependymal nodules establishing the diagnosis. Fetal MRI in the appropriate clinical setting can be potentially invaluable and can have important prognostic implications.
\end{abstract}

Key words: antenatal diagnosis; fetus; tuberous sclerosis; ultrafast MRI; ultrasound.

Tuberous sclerosis (TS) is an autosomal dominant disorder with variable expressivity and high penetrance characterized by multi-organ hamartomas. The classical clinical triad of seizures, mental retardation and adenoma sebaceum (Vogt), replaced by Gomez-Roach criteria, further modified in 1998, ${ }^{[1]}$ more accurately account for clinicopathological heterogeneity of this condition. Seventy percent of cases are sporadic with new mutations in TSC1/9q34 or TSC2/16p13 genes. ${ }^{[2]}$

Previously, the antenatal diagnosis of TS could only be suggested by positive family history and detection of cardiac rhabdomyomas. Currently, primary and adjunctive imaging modalities such as ultrasound and antenatal MRI are becoming indispensable, as described in this and other reports. ${ }^{[1-4]}$

\section{Case report}

Routine ultrasound in a 28-year-old primigravida at 26 weeks revealed multiple echodense cardiac masses suggesting rhabdomyomas and high possibility of TS, despite a lack of family history. Brain and other fetal organs were normal.
Antenatal MRI was performed with a $1.5 \mathrm{~T}$ unit (Signa Echospeed, General Electric, Milwaukee, USA). A six-element phased-array torso coil was used without maternal sedation or fetal curarization. Multiplanar Single Shot Fast Spin Echo (SSFSE) sequences were obtained [section thickness $5 \mathrm{~mm}$, Field Of View (FOV) 35 x $35 \mathrm{~cm}$, acquisition matrix $256 \mathrm{x}$ 256 and acquisition time $18 \mathrm{~s}$ ]. Triplanar (axial, sagittal and coronal), orthogonal images of the gravid uterus and fetal head were obtained with each sequence serving as a scout for the subsequent one.

Magnetic Resonance Imaging revealed focal hypointensities along the wall of the right lateral ventricle [Figures 1 and 2], indicative of subependymal nodules. A $9 \mathrm{~mm}$ sized area, hypointense to white matter, in the left frontal cortex [Figures 2 and 3], suggested a cortical tuber. All lesions were confirmed on three planes. Intracardiac tumors appeared hyperintense. Using Roach's criteria, a diagnosis of TS was made and the patient counseled. Considering social implications, delivery was induced and findings were confirmed at autopsy.

\section{Discussion}

Incidence of TS is $1: 10$ 000-1 : 50 000. A forme fruste of the disease is probably frequent. ${ }^{[5]}$ It has variable expressivity with functional prognosis related to neurological outcome (seizure potential and high rate of mental retardation). TSC1 mutations are rarer and milder than TSC2 mutations, having fewer cortical and subependymal lesions. Clinical features correlate better with renal angiomyolipomas than tuber count. ${ }^{[5]}$

Cardiac rhabdomyomas, especially when multiple, are strongly associated with TS although diagnosis (and implications) of TS remain uncertain. Prognosis of isolated rhabdomyomas is usually good. Eighty-seven percent of fetuses with cardiac rhabdomyomas have TS, ${ }^{[4]}$ while $50 \%$ of TS have cardiac rhabdomyomas. ${ }^{[6]}$

Prenatal assessment plays a vital role in management of TS 


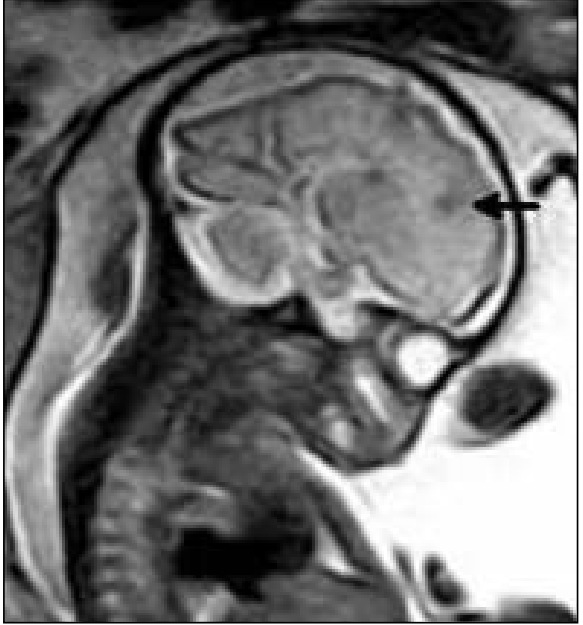

Figure 1: Left parasagittal image (SSFSE) showing a hypointense focus in a subependymal location involving the left lateral ventricle (arrow)

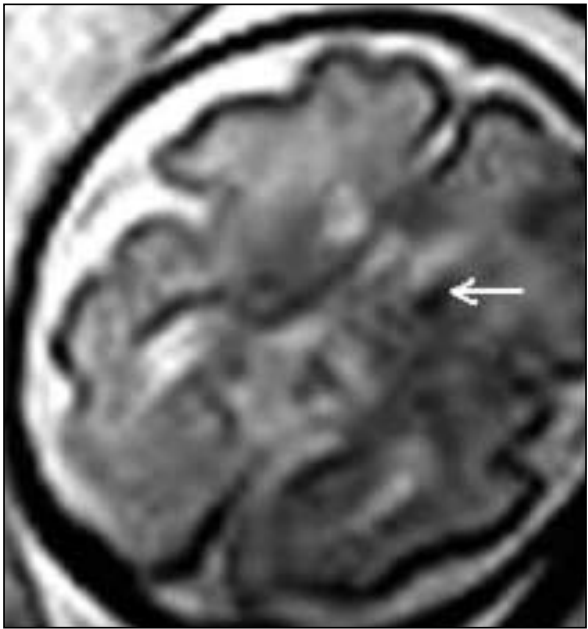

Figure 2: Axial SSFSE image at the midventricular level showing a hypointense area in the left frontal cortex representing a cortical tuber along with subependymal nodules involving the lateral ventricle (arrows)

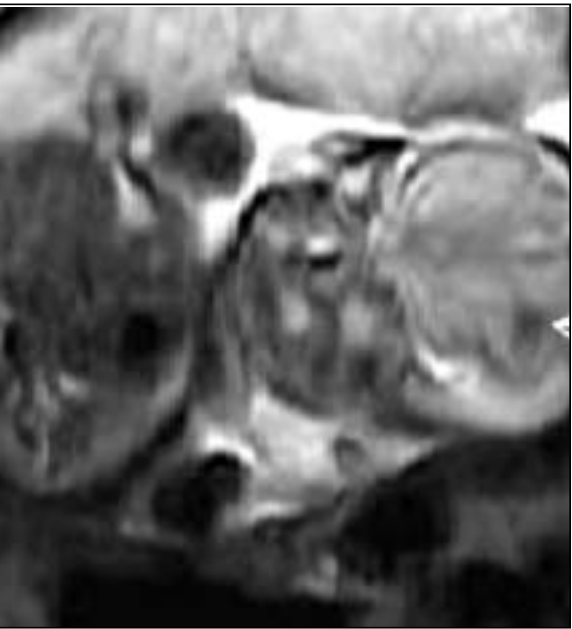

Figure 3: Coronal SSFSE image at the level of the frontal horn depicting the cortical tuber in the left frontal cortex (arrow) and serves as prognostic indicator for parents and genetic counselors. Despite genetic tests, $30 \%$ of TS patients have negative results. ${ }^{[5]}$

Ultrasound has been the primary modality for fetal assessment but is limited for brain evaluation. As in our patient, intracardiac tumors appear echodense on ultrasound. ${ }^{[6]}$

Previously, antenatal MRI was unsuitable due to long scan times. Currently, faster sequences permit rapid acquisitions between fetal movements as also sequence repetition if required. Detection of subtle pathology like tubers has been possible as early as 21 weeks, ${ }^{[2]}$ due to high-spatiotemporal resolution, multiplanar capabilities and superior soft-tissue characterization of MRI. It allows a wider FOV, permitting more complete fetal evaluation. Consequently, it helps determine such diagnoses and prognoses, as $90 \%$ of patients have seizures and developmental delay. This diagnostic and prognostic void can thus be filled.

Hamartomas in TS can develop at any time. A negative prenatal imaging evaluation of the fetal CNS does not guarantee that hamartomas will not develop in the future.

Although a larger study is warranted, our report exemplifies the possible impact of MRI as a problem-solving tool. The diagnosis of TS and depiction of CNS pathology allows for accurate assessment. Decisions based solely upon imaging findings can possibly be made, enabling rational clinical management. As experience with antenatal MRI increases, its role and indications will be defined better. What stands beyond question, however, is its present-day utility in providing important fetal structural detail.

\section{References}

1. Roach ES, Gomez MR, Northrup H. Tuberous sclerosis complex consensus conference: revised clinical diagnostic eriteria. J Child Neurol 1998;13:624-8.

2. Levine D, Barnes P, Korf B, Edelman R. Tuberous sclerosis in the fetus: second-trimester diagnosis of subependymal tubers with ultrafast MR imaging. A.JR Am J Roentgenol 2000;175:1067-9

3. Chen CP, Liu YP, Huang JK, Chang TY, Chen MR, Chiu NC, et al. Contribution of ultrafast magnetic resonance imaging in prenatal diagnosis of sonographically undetected cerebral tuberous sclerosis associated with cardiac rhabdomyomas. Prenat Diagn 2005;25:523-4.

4. Sonigo P, Elmaleh A, Fennont L, Delezoide AL, Mirlesse V, Brunelle F. Prenatal MRI diagnosis of fetal tuberous sclerosis. Pediatr Radiol 1996;26:1-4

5. Dabora SL, Jozwiak S, Franz DN, Roberts PS, Nieto A, Chung J, et al. Mutational analysis in a cohort of 224 tuberous sclerosis patients indicates increased severity of TSC2, compared with TSC1, disease in multiple organs. Am .J Hum Genet 2001;68:64-80.

6. Gamzu R, Achiron R, Hegesh J, Weiner E, Tepper R, Nir A, et al. Evaluating the risk of tuberous sclerosis in cases with prenatal diagnosis of cardiac rhabdomyoma. Prenat Diagn 2002;22:1044-7.

Accepted on 30-07-2005 